University of Wollongong

Research Online

Faculty of Business - Papers (Archive)

Faculty of Business and Law

$1-1-2010$

\title{
Endogenous technological progress in a multi-sector growth model
}

Davide La Torre

Universita degli Studi di Milano

Simone Marsiglio

University of Wollongong, simonem@uow.edu.au

Follow this and additional works at: https://ro.uow.edu.au/buspapers

Part of the Business Commons

Research Online is the open access institutional repository for the University of Wollongong. For further information contact the UOW Library: research-pubs@uow.edu.au 


\title{
Endogenous technological progress in a multi-sector growth model
}

\begin{abstract}
This paper presents an endogenous growth model driven by human capital, where human capital can be allocated across three sectors: the production of the final consumption good, the educational sector and the production of technological capital (in the form of knowledge or ideas). In our model, which also includes public expenditure and population growth, labor augmenting technical progress is endogenous and this enriches the transitional dynamics of the economy. With respect to ideas-based growth models, we assume knowledge is produced according to a neoclassical technology, combining ideas and human capital. Such an assumption is motivated by empirical works showing the existence of significant decreasing returns in the creation of ideas at the aggregate level (as Kortum, 1993; and Pessoa, 2005) and of the weak relationship between some inputs of the knowledge production process (as the number of researchers) and the total factor productivity growth rate (as Jones, 2002). Under some general conditions, this economy exhibits the existence of a steady state equilibrium and an unstable multidimensional manifold. Numerical examples are provided to show the existence of stable arms.
\end{abstract}

\section{Keywords}

endogenous, technological, model, progress, multi, sector, growth

Disciplines

Business

\section{Publication Details}

La Torre, D. \& Marsiglio, S. (2010). Endogenous technological progress in a multi-sector growth model. Economic Modelling, 27 (5), 1017-1028. 


\title{
Endogenous Technological Progress in a Multi-Sector Growth Model*
}

\author{
Davide La Torre Simone Marsiglio $^{\ddagger}$ \\ Forthcoming in Economic Modelling
}

\begin{abstract}
This paper presents an endogenous growth model driven by human capital, where human capital can be allocated across three sectors: the production of the final consumption good, the educational sector and the production of technological capital (in the form of knowledge or ideas). In our model, which also includes public expenditure and population growth, labor augmenting technical progress is endogenous and this enriches the transitional dynamics of the economy. With respect to ideas-based growth models, we assume knowledge is produced according to a neoclassical technology, combining ideas and human capital. Such an assumption is motivated by empirical works showing the existence of significant decreasing returns in the creation of ideas at the aggregate level (as Kortum, 1993; and Pessoa, 2005) and of weak relationship between some inputs of the knowledge production process (as the number of researchers) and the total factor productivity growth rate (as Jones, 2002). Under some general conditions, this economy exhibits the existence of a steady state equilibrium and an unstable multidimensional manifold. Numerical examples are provided to show the existence of stable arms.
\end{abstract}

Keywords: Economic Growth, Physical and Human Capital Accumulation, Technological Progress JEL Classification: O33, O40, O41

\section{Introduction}

During the last century, modern economies have been characterized by growth in many forms: output, productivity, average human capital and knowledge are all variables which have grown consistently over the past hundred years. This increased the interest in identifying the underlying causes of such improvements, and different variables have been identified as possible sources of growth in the literature during the last fifty years. Solow (1965) and others after him identified the accumulation of physical capital as crucial in explaining growing economies. In Lucas' (1988) view, instead, the evolution of human capital is the key feature driving growth in the long run. Recently, a literature stream, led mainly by Romer (1986, 1990), has started considering ideas as the relevant engine of growth.

In ideas-based growth models, the dynamics of knowledge is assumed to be the ultimate determinant of growth. This means that the creation of new ideas is enough to explain the features of growing economies over the last century. In order to model this, some kind of linearities have been introduced in the technology production function (see Jones, 2005, for a survey). For example, Romer (1990) assumes creation of ideas is linear both in the stock of ideas and human capital employed in research. Empirical works suggest there are significant decreasing returns of ideas at the aggregate level (see for example, Kortum, 1993; and Pessoa, 2005). Moreover, as Pessoa (2005) clearly summarizes: "The ideas-driven model ... predicts that

${ }^{*}$ We are grateful to the anonymous referees for useful comments. This paper has benefitted from conversation on this subject with several colleagues. In particular, we thank Prof. H.Kunze for useful comments and suggestions.

${ }^{\dagger}$ University of Milan, Department of Economics, via Conservatorio 7, I-20122 Milan, Italy; davide.latorre@unimi.it

${ }^{\ddagger}$ University of Milan, Department of Economics, via Conservatorio 7, I-20122 Milan, Italy; simone.marsiglio@unimi.it 
expansion in the number of researchers leads to a permanent increase in TFP growth rate. In contrast, the empirical evidence suggests that most OECD economies have increased the size of their RED workforce, while experiencing (at best) constant TFP growth rates. This weak relationship between the number of researchers and TFP growth rate has led some to question the viability of ideas-driven growth for the long run". Therefore, we consider that the concept of creation of ideas has to be introduced in standard economic growth models, modeling its interaction with capital (in particular with human capital), in order to deepen their dynamics. In this paper, we introduce ideas in a multi-sector endogenous growth model. In particular, ideas affect the production of the consumption good and are used to create new ideas 1 . Therefore, our model economy is composed of three main interrelated sectors: the final one, the educational sector and that devoted to accumulating knowledge. We also include other two important variables: public expenditure and population growth (see also Hall (1988), Jorgenson and others (1987), Maddison (1982), Mehra and Prescott (1985)). The basic model we use in order to study the linkages among ideas, human and physical capital is an extension of the Uzawa-Lucas model (see Uzawa, 1965, and Lucas, 1988), which is one of the most studied and celebrated endogenous growth models. As Boucekkine and Ruiz-Tamarit (2008a) have recently emphasized, "the Lucas-Uzawa model, one of the most celebrated endogenous growth model, [...] has some interesting properties. [...] being a two-sector model, it gives rise to a sophisticated dynamic system with two controls, consumption (c) and the fraction of human capital operating in the final good sector (u), and two state variables, physical capital $(K)$ and human capital $(H)$ ", and "because it is mathematically appealing, it has been studied by many authors, using different approaches, allowing for a stimulating methodological discussion". In literature, further extensions of the classical Uzawa-Lucas model can be found in Boucekkine and others (2008b and 2009b), Bucci and others (2009, 2010a, 2010b and 2010c), Ferrara and Guerrini (2008 and 2010). For instance, in Bucci and others (2010c), the authors generalize the Uzawa-Lucas model by assuming that the level of technology might be subject to random shocks and suppose that the level of technology follows an exogenous stochastic differential equation driven by a Brownian motion. For a certain combination of the parameters and by using the Bellman equation, the authors find a closed-form solution to this model. In Bucci and others (2010b), the authors extend the Lucas-Uzawa model along two different directions, by introducing the growth of the physical capital stock into the human capital supply equation and by including in the intertemporal maximization problem of the representative household a preference parameter controlling for the degree of agents' altruism towards future generations.

In this paper we extend the Uzawa-Lucas model in different ways. Ideas affect the production of the final consumption good (derived from a technology combining ideas, physical and human capital) and are used to produce new ideas (therefore we have an additional differential equation, representing the accumulation of knowledge over time). Human capital, which still represents the engine of growth, is used also in the production of new ideas, and therefore has to be endogenously allocated across these three sectors.

It is well known that economic growth can be affected by government's purchases of goods and services. Therefore, we introduce public expenditure in our model and, following Barro R.J. (1990), Barro R.J. and Lee J.W. (2000) and Barro R.J. and Sala-i-Martin X. (1992), we assume that it is a fixed portion of the total income. We also assume that population grows at a variable rate and that it follows a logistic behavior. A benevolent social planner in this model has to decide where to allocate resources. The human capital

\footnotetext{
${ }^{1}$ The introduction of ideas in a growth model, as Jones (2005) emphasizes, raises the problem of introducing non-rivalry. Romer (1993) divides goods into two categories: ideas and objects. Ideas can be represented by instructions or blueprints, while objects are the standard rival goods, such as capital, labor, output... Ideas are merely instructions for combining the objects in order to produce utility. Accepting Romer's (1993) definition of ideas, we have to recognize that the use of ideas by one person does not diminish others' use and therefore ideas are non-rival goods. This of course implies the presence of increasing returns to scale in production possibilities, and consequently leads to a framework where the first fundamental theorem of welfare economics does not hold. As a result, the decentralized outcome may not coincide with the planned one, resulting in a sub optimal allocation of resources. In this paper, we do not analyze the problems related to the decentralized allocation (postponing this issue to future research), but we aim to study the optimal planned economy and the linkages between ideas, physical and human capital
} 
decision is crucial, since it affects production of the final good, education, and ideas.

The paper is organized as follows. Section 2 introduces the model in its most possible general version. It shows that this model is equivalent to a much simpler version simply by rescaling some variables, and then derives the optimal paths. Section 3 focuses on a particular case of the model, namely when the production function of new ideas shows constant returns to scale. It analyzes the steady state of our model economy, that is characterized by a balanced growth path, along which the fractions of human capital allocated in each sector are constant, and its local transitional dynamics. We show that the economy exhibits a steady state equilibrium and an unstable multidimensional manifold which has at least dimension one. Numerical examples are provided to show the existence of stable arms which prove that this equilibrium is a saddle point. We moreover present a numerical example, in order to underline the role of human capital in the three main sectors. As usual, section 4 concludes.

\section{The Model}

The model is an extension of the Uzawa-Lucas model, where we consider a multi-sector economy, where all variables are used to produce only one homogeneous final good, that can be consumed or invested in physical capital. Physical capital can be used only for producing the final good, while the human capital can be allocated in the production of the final good, in the education sector or in the production of ideas. Technological capital or ideas instead is used as an input in the production of the final good or to create ideas. The economy is closed and composed of households (that receive wages and interest income, purchase the consumption good and choose how much to save and how much to invest both in education and in ideas), firms (that produce the consumption good) and government (which finances its expenditures through lumpsum taxes). The infinitely-lived representative household wants to maximize its lifetime utility function:

$$
U=\int_{0}^{\infty} u\left(c_{t}\right) L_{t} e^{-\rho t} d t
$$

where $c_{t} \equiv \frac{C_{t}}{L_{t}}$ is per-capita consumption and $\rho>0$ is the rate of time preference. The instantaneous utility function is assumed to be iso-elastic:

$$
u\left(c_{t}\right)=\frac{c_{t}^{1-\sigma}}{1-\sigma}
$$

where $\sigma>0$ represents the inverse of the inter-temporal elasticity of substitution in consumption.

Following Barro and Sala-i-Martin (1992), we assume that (not wasteful) public expenditure, $G_{t}$, is a fixed portion $\tau \in[0,1]$ of the income $Y_{t}$ for all $t$, that is:

$$
G_{t}=\tau Y_{t}
$$

In literature, it is well recognized that economic growth can be affected by government's purchases of goods and services. Therefore, we introduce government in our model in order to have the most possible general model. However, as we show in the next subsection, the presence of government spending is irrelevant for the equilibrium of the model. In fact, with a simple transformation of variables it is possible to recast the model in a simpler form, where government activity is no longer present.

The final good is produced by combining physical capital, $K_{t}$, the share of human capital allocated to final production, $u_{t} H_{t}$, labor in efficiency units (the product of labor and its efficiency, given by level of ideas in the economy), $A_{t} L_{t}$, and public expenditure $G_{t}$ according to the following technology (see Mankiw et al. (1992)):

$$
Y(t)=\left(A_{t} L_{t}\right)^{\psi_{1}}\left(u_{t} H_{t}\right)^{\psi_{2}} K_{t}^{\psi_{3}} G_{t}^{1-\psi_{1}-\psi_{2}-\psi_{3}},
$$

with $\psi_{1}, \psi_{2}, \psi_{3} \in(0,1)$ and $u_{t} \in(0,1)$. 


\begin{tabular}{|c|c|c|}
\hline & $\eta_{1}$ & $\eta_{2}$ \\
\hline USA & 0.03857713 & -0.00000041 \\
\hline Italy & 0.10548241 & -0.00000776 \\
\hline UK & 0.15565694 & -0.00000869 \\
\hline
\end{tabular}

Table 1: Estimated parameters

Notice that two inputs of production, human capital and ideas, are allocated across other sectors: other than the final one, the former is also assigned to production of new human capital and production of new ideas, while the latter is also assigned to the creation of knowledge. The main difference between these factors is found in their own nature: human capital is a rival good, meaning that its usage in a sector lowers its availability in the other ones 2 . This means that human capital used in the production of the final good is only a share, $u_{t}$, of the total, while the remaining fraction is split between the production of new ideas (or blueprints), $x_{t}$ and education, $1-u_{t}-x_{t}$.

Population (raw labor) coincides with the available number of workers, $L_{t}$, so that there is no unemployment and the labor supply is inelastic (no leisure-work choice), and it grows at an exogenous rate. We assume that population grows at a variable rate $g\left(L_{t}\right)=\eta_{1}-\eta_{2} L_{t}, \eta_{i}>0, \eta_{1}-\eta_{2} L_{0}>0$, that is it follows a logistic behavior ${ }^{3}$. The first study on population dynamics was due to Malthus (1798), who showed the explosion of the birth rate when income increases and then the increase of mortality because of competition on the relatively scarce output of productive land. Malthus (1798) was among the first to point out the existence of these two distinct phases in the evolution of world population. This idea was formalized by a logistic process by Verhulst (1977), and by the famous papers by Lotka (1925) and Volterra (1931). Applied mathematicians and biologists have studied extensively the dynamics of populations using logistic processes and their generalizations. Up-to-date demographic forecasts (United Nations, 2000) show that the world population annual growth rate is expected to fall gradually from $1.8 \%$ (1950-2000) to $0.9 \%$ (2000-2050) and then reach a value of $0.2 \%$ between the years 2050 and 2100 . In Capasso and others (2010b), an estimation of $\eta_{1}$ and $\eta_{2}$ is provided. Starting from real data (coming from Angus Maddison webpage: http://www.ggdc.net/Maddison/dataset) we solve an inverse problem for the differential equation $\dot{L}_{t}=L_{t} g\left(L_{t}\right)$. This empirical analysis shows that a good fitting curve for this data is the logistic one; the results to eight decimal digits in three countries (Italy, UK, USA) over the period 1870-2008 are shown in Table 1 ,
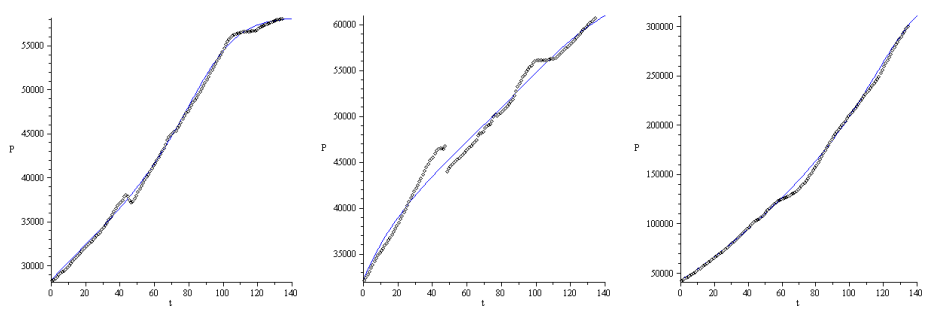

Figure 1: Population dynamics in Italy, USA, UK

Physical capital accumulates over time in accordance to the difference between output, $Y_{t}$, consumption, $C_{t}$ and public expenditure $G_{t}$ :

$$
\dot{K}_{t}=Y_{t}-C_{t}-G_{t} .
$$

\footnotetext{
${ }^{2}$ Ideas instead are non-rival, meaning that being widespread in the economy, they can be freely and contemporaneously used in different sectors, without compromising their availability in each one.

${ }^{3}$ If we assume that $\eta_{2}=0$ this implies that population evolves over time according to an exponential process
} 
Human capital accumulation coincides with the production of new human capital, which depends only on the effort devoted to the accumulation of human capital, $1-u_{t}-x_{t}$, and on the already attained human capital stock (the education sector is intensive in human capital), $H_{t}$ :

$$
\dot{H}_{t}=B\left(1-u_{t}-x_{t}\right) H_{t},
$$

where $0<u_{t}, x_{t}<1$ and $B>0$ is a scale parameter. Notice that new human capital is produced accordingly to a linear production function, meaning that human capital, as in the Uzawa-Lucas model, represents the force driving endogenous growth.

Also idea accumulation coincides with the production of new ideas, depending on the effort devoted to the accumulation of new ideas, $x_{t} H_{t}$, and on already existing stock of the knowledge, $A_{t}$ :

$$
\dot{A}_{t}=D\left(x_{t} H_{t}\right)^{\phi} A_{t}^{\delta},
$$

where $\phi \in[0,1), \delta \in[0,1)$ (see Lutz (1998)) and $D>0$ is a scale parameter. Let us observe that if $\phi=0$ and $\delta=1$ the technological progress is exogenous and this model reduces to the deterministic version presented in Bucci and others (2010c). If $\delta=0$ this equation is trivial. The condition $\delta>0$ means we rely on what Jones (2005) labels as a standing on the shoulders effect: the discovery of ideas in the past increases the possibilities of new discoveriest As in Jones (2005), we assume that the production of new ideas is Cobb-Douglas, combining ideas and human capital. The specification of decreasing returns in ideas is suggested also in Romer (1990) and considered by Kortum (1993) and Jones (1995). Moreover, empirical works suggest there are significant decreasing returns of ideas at the aggregate level; for example, Kortum (1993) reports elasticity estimates of $\delta$ in the range between 0.1 and 0.6. Romer (1990) also suggests the specification of decreasing returns in human capital, considered by Stokey (1995) and Jones (2002).

In next section, in order to provide the existence of a BGP equilibrium and sufficiency of the Hamiltonianbased optimality condition 5 we will assume that $\delta=1-\phi$. If this hypothesis is not assumed, one has to apply a suitable transformation of variables to guarantee this property (see Hiraguchi (2009a and 2009b) and Bucci and others (2010a)) being satisfied. Following Kortum (1993) estimates, the assumption of constant returns to scale implies that $\phi$ lies in the interval of 0.4 to 0.9 . Notice that in such a case we are considering the possibility of duplication in technology: if we double the stock of ideas and the share of human capital used for producing new ideas, we will also double the production of new knowledge. However, in order to do so, we have to allow a decrease in the fraction of human capital allocated to the educational sector, meaning that the endogenous growth rate will be lower.

We have assumed that the depreciation rate of physical, human and technological capital is common and equal to zerd ${ }^{6}$.

The social planner maximizes the social welfare function, that is it has to choose $c_{t}, u_{t}$, and $x_{t}$ in order to maximize agents' lifetime utility function subject to physical, human capital and idea accumulation constraints and the initial conditions:

$$
\begin{aligned}
\max _{\left\{c_{t}, u_{t}, x_{t}\right\}_{0}^{\infty}} & U=\int_{0}^{\infty} \frac{c_{t}^{1-\sigma}}{1-\sigma} L_{t} e^{-\rho t} d t, \rho>0 \\
\text { s.t. } & \dot{K}_{t}=\left(A_{t} L_{t}\right)^{\psi_{1}}\left(u_{t} H_{t}\right)^{\psi_{2}} K_{t}^{\psi_{3}} G_{t}^{1-\psi_{1}-\psi_{2}-\psi_{3}}-c_{t} L_{t}-G_{t} \\
& \dot{H}_{t}=B\left(1-u_{t}-x_{t}\right) H_{t} \\
& \dot{A}_{t}=D\left(x_{t} H_{t}\right)^{\phi} A_{t}^{\delta}
\end{aligned}
$$

\footnotetext{
${ }^{4}$ The opposite case, where $\delta<0$, corresponds to what the productivity literature defines as the fishing out effect, in which the rate of innovation decreases with the level of knowledge

${ }^{5}$ As Hiraguchi (2009) underlines, the Hamiltonian is nonconcave and thus the first order conditions (FOCs) and the transversality conditions does not imply the optimality.

${ }^{6}$ This is of course a simplifying assumption, but the outcome of the model would not change even if we introduce non zero depreciation rates. The only difference would be given by the presence of additional constants in the Euler and state equations
} 


$$
\begin{aligned}
& \dot{L}_{t}=g\left(L_{t}\right) L_{t} \\
& G_{t}=\tau Y_{t} \\
& K_{0}, H_{0}, A_{0}, L_{0} \text { given. }
\end{aligned}
$$

At the end of this section, it is worth mentioning how one can obtain estimates of parameters for this optimal control problem using, for instance, fractal-based methods and inverse problem approach. In fact, in practical applications it is crucial to obtain such estimates of meaningful parameters starting from a set of observational data. As well discussed in Bucci and others (2010b) and Kunze and La Torre (2009b), through the solution of the inverse problem one can get the estimation of some key-parameters of the model, such as the total factor productivity, the productivity of human capital in the production of new skills, the physical capital share in total income, and so on. The method we use is based on the Collage Theorem; many problems in the parameter estimation literature for differential equations can be formulated in a collage coding framework as showed in Kunze and Vrscay (1999) and subsequent works. The main advantage of this approach, motivated by its abstract formulation, is that it can be used whenever classical econometric techniques fail (see, for more details, Kunze and others (1999), (2003), (2004), (2007a), (2007b), (2007c), (2009a), (2009b) in the references).

\subsection{Solving the model}

By combining the expression $G_{t}=\tau Y_{t}$ and the definition of production function $Y$ we get

$$
Y_{t}=\left(A_{t} L_{t}\right)^{\frac{\psi_{1}}{\psi_{1}+\psi_{2}+\psi_{3}}}\left(u_{t} H_{t}\right)^{\frac{\psi_{2}}{\psi_{1}+\psi_{2}+\psi_{3}}} K_{t}^{\frac{\psi_{3}}{\psi_{1}+\psi_{2}+\psi_{3}}} \tau^{\frac{1-\psi_{1}-\psi_{2}-\psi_{3}}{\psi_{1}+\psi_{2}+\psi_{3}}}
$$

This means we can eliminate the variable $G_{t}$ and, by defining $\alpha=\frac{\psi_{3}}{\psi_{1}+\psi_{2}+\psi_{3}}, \beta=\frac{\psi_{2}}{\psi_{1}+\psi_{2}+\psi_{3}}$ and $\Omega=$ $\tau^{\frac{1-\psi_{1}-\psi_{2}-\psi_{3}}{\psi_{1}+\psi_{2}+\psi_{3}}}$, the model can be rewritten as

$$
\begin{aligned}
\max _{\left\{c_{t}, u_{t}, x_{t}\right\}_{0}^{\infty}} & U=\int_{0}^{\infty} \frac{c_{t}^{1-\sigma}}{1-\sigma} L_{t} e^{-\rho t} d t, \rho>0 \\
\text { s.t. } & \dot{K}_{t}=\Omega K_{t}^{\alpha}\left(u_{t} H_{t}\right)^{\beta}\left(A_{t} L_{t}\right)^{1-\alpha-\beta}-c_{t} L_{t} \\
& \dot{H}_{t}=B\left(1-u_{t}-x_{t}\right) H_{t} \\
& \dot{A}_{t}=D\left(x_{t} H_{t}\right)^{\phi} A_{t}^{\delta} \\
& \dot{L}_{t}=g\left(L_{t}\right) L_{t} \\
& K_{0}, H_{0}, A_{0}, L_{0} \text { given. }
\end{aligned}
$$

Furthermore by applying a suitable transformation of variables the model can be rewritten as

$$
\begin{aligned}
\max _{\left\{c_{t}, u_{t}, x_{t}\right\}_{0}^{\infty}} & U=\int_{0}^{\infty} \frac{c_{t}^{1-\sigma}}{1-\sigma} L_{t} e^{-\rho t} d t, \rho>0 \\
\text { s.t. } & \dot{K}_{t}=\Omega K_{t}^{\alpha}\left(u_{t} H_{t}\right)^{\beta}\left(A_{t} L_{t}\right)^{1-\alpha-\beta}-c_{t} L_{t} \\
& \dot{H}_{t}=\left(1-u_{t}-x_{t}\right) H_{t} \\
& \dot{A}_{t}=\left(x_{t} H_{t}\right)^{\phi} A_{t}^{\delta} \\
& \dot{L}_{t}=g\left(L_{t}\right) L_{t} \\
& K_{0}, H_{0}, A_{0}, L_{0} \text { given. }
\end{aligned}
$$

The Hamiltonian function, $\mathcal{H}_{t}(\cdot)$, associated to the maximization problem (since $L_{t}$ is exogenous) is:

$$
\mathcal{H}_{t}(\cdot)=\frac{c_{t}^{1-\sigma}}{1-\sigma} L_{t} e^{-\rho t}+\lambda_{t}\left[\Omega K_{t}^{\alpha}\left(u_{t} H_{t}\right)^{\beta}\left(A_{t} L_{t}\right)^{1-\alpha-\beta}-c_{t} L_{t}\right]+\mu_{t}\left[\left(x_{t} H_{t}\right)^{\phi}\left(A_{t} L_{t}\right)^{\delta}\right]+\nu_{t}\left(1-u_{t}-x_{t}\right) H_{t} .
$$


The first order necessary conditions are:

$$
\begin{aligned}
c_{t} & \rightarrow c_{t}^{-\sigma} e^{-\rho t}=\lambda_{t} \\
u_{t} & \rightarrow \lambda_{t} \beta \Omega K_{t}^{\alpha}\left(u_{t} H_{t}\right)^{\beta-1}\left(A_{t} L_{t}\right)^{1-\alpha-\beta} H_{t}=\nu_{t} H_{t} \\
x_{t} & \rightarrow \mu_{t} \phi\left(x_{t} H_{t}\right)^{\phi-1} A_{t}^{\delta} H_{t}=\nu_{t} H_{t} \\
K_{t} & \rightarrow-\dot{\lambda}_{t}=\lambda_{t} \Omega \alpha K_{t}^{\alpha-1}\left(u_{t} H_{t}\right)^{\beta}\left(A_{t} L_{t}\right)^{1-\alpha-\beta} \\
A_{t} & \rightarrow-\dot{\mu}_{t}=\lambda_{t}(1-\alpha-\beta) \Omega K_{t}^{\alpha}\left(u_{t} H_{t}\right)^{\beta}\left(A_{t} L_{t}\right)^{-\alpha-\beta}+\mu_{t} \delta\left(x_{t} H_{t}\right)^{\phi} A_{t}^{\delta-1} \\
H_{t} & \rightarrow-\dot{\nu}_{t}=\lambda_{t} \beta \Omega K_{t}^{\alpha}\left(u_{t} H_{t}\right)^{\beta-1}\left(A_{t} L_{t}\right)^{1-\alpha-\beta} u_{t}+\mu_{t} \phi\left(x_{t} H_{t}\right)^{\phi-1} A_{t}^{\delta} x_{t}+\nu_{t}\left(1-u_{t}-x_{t}\right)
\end{aligned}
$$

together with the initial conditions $K_{0}, H_{0}$ and $A_{0}$, the dynamic constraints:

$$
\begin{aligned}
\dot{K}_{t} & =\Omega K_{t}^{\alpha}\left(u_{t} H_{t}\right)^{\beta}\left(A_{t} L_{t}\right)^{1-\alpha-\beta}-c_{t} L_{t} \\
\dot{H}_{t} & =\left(1-u_{t}-x_{t}\right) H_{t} \\
\dot{A}_{t} & =\left(x_{t} H_{t}\right)^{\phi} A_{t}^{\delta}
\end{aligned}
$$

and the transversality conditions:

$$
\begin{array}{ll}
\lim _{t \rightarrow \infty} & \lambda_{t} K_{t}=0 \\
\lim _{t \rightarrow \infty} & \nu_{t} H_{t}=0 \\
\lim _{t \rightarrow \infty} & \mu_{t} A_{t}=0 .
\end{array}
$$

Solving the system, the optimal paths of consumption, share of human capital allocated to production and to new ideas are:

$$
\begin{aligned}
\frac{\dot{c_{t}}}{c_{t}} & =\frac{1}{\sigma}\left[\alpha \Omega K_{t}^{\alpha-1}\left(u_{t} H_{t}\right)^{\beta}\left(A_{t} L_{t}\right)^{1-\alpha-\beta}-\rho\right] \\
\frac{\dot{u_{t}}}{u_{t}} & =\frac{1}{\beta-1}\left[\alpha \frac{c_{t} L_{t}}{K_{t}}-\beta-(1-\beta)\left(u_{t}+x_{t}\right)-(1-\alpha-\beta)\left(x_{t} H_{t}\right)^{\phi} A_{t}^{\delta-1}\right] \\
\frac{\dot{x_{t}}}{x_{t}} & =\frac{1}{\phi-1}\left[\frac{\phi(1-\alpha-\beta)}{\beta} u_{t} x_{t}^{\phi-1} H_{t}^{\phi} A_{t}^{\delta-1}-\phi-(1-\phi)\left(u_{t}+x_{t}\right)\right]
\end{aligned}
$$

Equation (22) is the standard Keynes-Ramsey rule, showing that the growth rate of consumption is an increasing function of the marginal productivity of physical capital in the final sector. Notice that, as usual, it is positive if the marginal productivity of physical capital is higher than the rate of time preference, $\rho$. Equation (23) says that the change of the share of human capital allocated to the production of the final good is a negative function of the consumption-capital ratio and a positive function of the average productivity of ideas in the research sector and of the shares of human capital not allocated to the educational sector. Equation (24) instead relates the share of human capital devoted to the production of ideas positively with the shares of human capital not allocated to the educational sector and with the $\frac{u_{t}}{x_{t}}$ ratio while negatively with the human capital-ideas ratio.

\section{$3 \quad$ Steady State Analysis}

We now analyze the steady state of our model economy, which is characterized by a balanced growth path equilibrium, that is a situation where all economic variables grow at constant and finite rates. Before doing this, we assume the following hypotheses: 
- $\delta=1-\phi$. Under this hypothesis the Hamiltonian is concave and thus the first order conditions (FOCs) and the transversality conditions imply the optimality (see Chilarescu (2008), Hiraguchi (2009a) and $(2009 b)) 7$

- $L_{t}$ is constant over time. Since we have assumed that $L_{t}$ follows a logistic behavior, in the long-run $L_{t}$ converges to a constant. Since, by continuity, nothing changes in the long-run perspective, then in the sequel it is worth assuming $L_{t}$ being constant over time.

By rescaling $L_{t}$ for simplicity, the previous model is totally equivalent to the following (where per-capita (lower case letters) and aggregate variables (capital letters) coincide):

$$
\begin{aligned}
\max _{\left\{c_{t}, u_{t}, x_{t}\right\}_{0}^{\infty}} & U=\int_{0}^{\infty} \frac{c_{t}^{1-\sigma}}{1-\sigma} e^{-\rho t} d t, \rho>0 \\
\text { s.t. } & \dot{k}_{t}=k_{t}^{\alpha}\left(u_{t} h_{t}\right)^{\beta} a_{t}^{1-\alpha-\beta}-c_{t} \\
& \dot{h}_{t}=\left(1-u_{t}-x_{t}\right) h_{t} \\
& \dot{a}_{t}=\left(x_{t} h_{t}\right)^{\phi} a_{t}^{1-\phi} \\
& k_{0}, h_{0}, a_{0} \text { given. }
\end{aligned}
$$

The following definition introduces the notion of BGP.

Definition 1: (Balanced Growth Path, BGP) a balanced growth path, BGP, or steady state equilibrium, $\left(\bar{c}, \bar{h}, \bar{k}, \bar{a}, \bar{u}, \bar{x}, \gamma_{c}, \gamma_{h}, \gamma_{k}, \gamma_{a}, \gamma_{u}, \gamma_{x}\right)$, is a sequence of time paths, $\left\{c_{t}, h_{t}, k_{t}, a_{t}, u_{t}, x_{t}\right\}_{t \geq 0}$, along which all economic variables grow at constant rates. A BGP is said to be non-degenerate if $c_{t}, h_{t}, k_{t}$ and $a_{t}$ grow at non negative rates.

First of all, notice that the growth rate of consumption must equalize that of physical capital, in order to have endogenous growth and not to violate TVC (19). Moreover, along the BGP, the share of human capital allocated in the production of the consumption good and in the creation of new ideas have to both be constant, otherwise the growth rate of human capital cannot be constant.

Therefore, we can study the dynamics of a simplified system, where the variables do not asymptotically grow, and the BGP of the original system is represented by the equilibrium point of such a simplified system. In fact, by introducing the intensive variables: $\chi_{t} \equiv \frac{c_{t}}{k_{t}}, \varphi_{t} \equiv \frac{h_{t}}{k_{t}}$ and $\psi_{t} \equiv \frac{a_{t}}{k_{t}}$, we obtain the following system of five nonlinear differential equations:

$$
\begin{aligned}
\frac{\dot{\chi_{t}}}{\chi_{t}} & =\frac{\alpha-\sigma}{\sigma}\left(u_{t} \varphi_{t}\right)^{\beta} \psi_{t}^{1-\alpha-\beta}-\frac{\rho}{\sigma}+\chi_{t} \\
\frac{\dot{\psi_{t}}}{\psi_{t}} & =\left(x_{t} \varphi_{t}\right)^{\phi} \psi_{t}^{-\phi}-\left(u_{t} \varphi_{t}\right)^{\beta} \psi_{t}^{1-\alpha-\beta}+\chi_{t} \\
\frac{\dot{\varphi_{t}}}{\varphi_{t}} & =\left(1-u_{t}-x_{t}\right)-\left(u_{t} \varphi_{t}\right)^{\beta} \psi_{t}^{1-\alpha-\beta}+\chi_{t} \\
\frac{\dot{u_{t}}}{u_{t}} & =\frac{1}{\beta-1}\left[\alpha \chi_{t}-\beta-(1-\beta)\left(u_{t}+x_{t}\right)-(1-\alpha-\beta)\left(x_{t} \varphi_{t}\right)^{\phi} \psi_{t}^{-\phi}\right] \\
\frac{\dot{x_{t}}}{x_{t}} & =\frac{1}{\phi-1}\left[\frac{\phi(1-\alpha-\beta)}{\beta} \frac{u_{t}}{x_{t}}\left(x_{t} \varphi_{t}\right)^{\phi} \psi_{t}^{-\phi}-\phi-(1-\phi)\left(u_{t}+x_{t}\right)\right] .
\end{aligned}
$$

\footnotetext{
${ }^{7}$ Through Comparison Theorems it is also possible to compare the dynamics of Equations (22), (23) and (24) with the case of constant returns to scale (see also Bucci and others (2010d)).
} 
Moreover, by introducing the variables $Z_{t}=\left(u_{t} \varphi_{t}\right)^{\beta} \psi_{t}^{1-\alpha-\beta}$ and $M_{t}=\left(x_{t} \varphi_{t}\right)^{\phi} \psi_{t}^{-\phi}$, we can recast the system in a quasi linear form:

$$
\begin{aligned}
\frac{\dot{\chi_{t}}}{\chi_{t}} & =\frac{\alpha-\sigma}{\sigma} Z_{t}-\frac{\rho}{\sigma}+\chi_{t} \\
\frac{\dot{Z}_{t}}{Z_{t}} & =\frac{\alpha+\beta-1}{\beta-1} \chi_{t}-\frac{\beta}{\beta-1}-(1-\alpha) Z_{t}-\frac{1-\alpha-\beta}{\beta-1} M_{t} \\
\frac{\dot{M_{t}}}{M_{t}} & =\frac{\phi^{2}(1-\alpha-\beta)}{\beta(\phi-1)} \frac{u_{t}}{x_{t}} M_{t}-\phi M_{t}-\frac{\phi}{\phi-1} \\
\frac{\dot{u_{t}}}{u_{t}} & =\frac{1}{\beta-1}\left[\alpha \chi_{t}-\beta-(1-\beta)\left(u_{t}+x_{t}\right)-(1-\alpha-\beta) M_{t}\right] \\
\frac{\dot{x_{t}}}{x_{t}} & =\frac{1}{\phi-1}\left[\frac{\phi(1-\alpha-\beta)}{\beta} \frac{u_{t}}{x_{t}} M_{t}-\phi-(1-\phi)\left(u_{t}+x_{t}\right)\right] .
\end{aligned}
$$

The equilibrium point of this system is represented by a point where equations (31), (32), (33), (34) and (35) are null. Such a point is characterized by a strictly positive level of all variables if $\sigma>1-\rho>0$. Moreover, under the same assumption, the equilibrium shows a multidimensional unstable manifold. Notice that such a parametric condition is usually satisfied: it only requires the inverse of the intertemporal elasticity of substitution to be sufficiently high. Several empirical works suggest the elasticity of substitution is lower than one, meaning that its inverse (the relative risk aversion parameter) is higher than one (see for example, Mehra and Prescott, 1985; and Hall, 1988). Therefore, we can summarize these results in the following propositions.

Proposition 1: if the following parameter restrictions apply:

$$
\sigma>1-\rho>0,
$$

then the following results hold:

(i) the BGP equilibrium is characterized by a strictly positive level of consumption, physical, human and technological capital, shares of human capital allocated to the educational and research sectors;

(ii) the BGP equilibrium shows a multidimensional unstable manifold.

Proof: see Appendixes A and B. Appendix A proves part (i) of the proposition while Appendix B part (ii).

Moreover, under standard parameter values, it is possible to show that the BGP equilibrium is saddlepoint stable. This is shown by the next numerical examples.

Example 3.1. Let us do a numerical simulation using the following parameter values: $\alpha=0.3333, \beta=0.2$, $\phi=0.2, \sigma=2, \rho=0.04$. The equilibrium is: $M^{*}=0.48, \chi^{*}=2.52, Z^{*}=3, u^{*}=0.3813, x^{*}=0.1386$. The linearized Jacobian is:

$$
\left[\begin{array}{ccccc}
2.520000000 & -2.100000000 & 0 & 0 & 0 \\
1.750000000 & -2.0 & 1.750000000 & 0 & 0 \\
0 & 0 & -0.2499999999 & -0.1938461539 & 0.5330769228 \\
-0.1588888888 & 0 & 0.2224444445 & 0.3813333333 & 0.3813333333 \\
0 & 0 & -0.2224444445 & -0.1413333333 & 0.9086666667
\end{array}\right]
$$

The eigenvalues of the linearized model are $\lambda_{1}=-.8982525480, \lambda_{2}=1.418252548, \lambda_{3}=.5199999998$, $\lambda_{4}=.6952597330, \lambda_{5}=-.1752597328$ with eigenvectors 


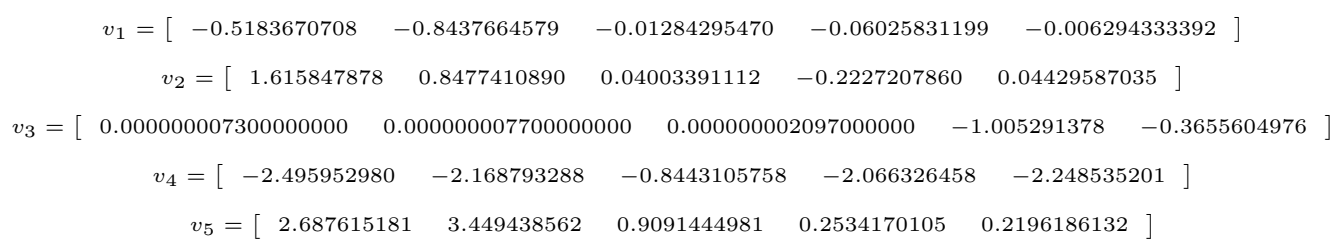

The unstable manifold $M_{u}$ is generated by the vectors $\left\langle v_{2}, v_{3}, v_{4}\right\rangle$ while the stable manifold $M_{s}$ by the vector $\left\langle v_{1}, v_{5}\right\rangle$.

Example 3.2. Let us do a numerical simulation using the following parameter values: $\alpha=0.3333, \beta=0.2$, $\phi=0.8, \sigma=2, \rho=0.04$. The equilibrium is: $M^{*}=0.48, \chi^{*}=2.52, Z^{*}=3, u^{*}=0.2611555556$, $x^{*}=0.2588444444$. The linearized Jacobian is:

$\left[\begin{array}{ccccc}2.520000000 & -2.100000000 & 0 & 0 & 0 \\ 1.750000000 & -2.0 & 1.750000000 & 0 & 0 \\ 0 & 0 & -4.000000003 & -6.646153850 & 6.705494512 \\ -0.1088148148 & 0 & 0.1523407408 & 0.2611555556 & 0.2611555556 \\ 0 & 0 & -2.437451853 & -4.221155557 & 4.778844445\end{array}\right]$

The eigenvalues of the linearized model are $\lambda_{1}=1.496661179+.6462436692 i, \lambda_{2}=1.496661179-.6462436692 i$, $\lambda_{3}=-.9766611807+.6462436678 i, \lambda_{4}=-.9766611807-.6462436678 i, \lambda_{5}=.5200000002$, with eigenvectors

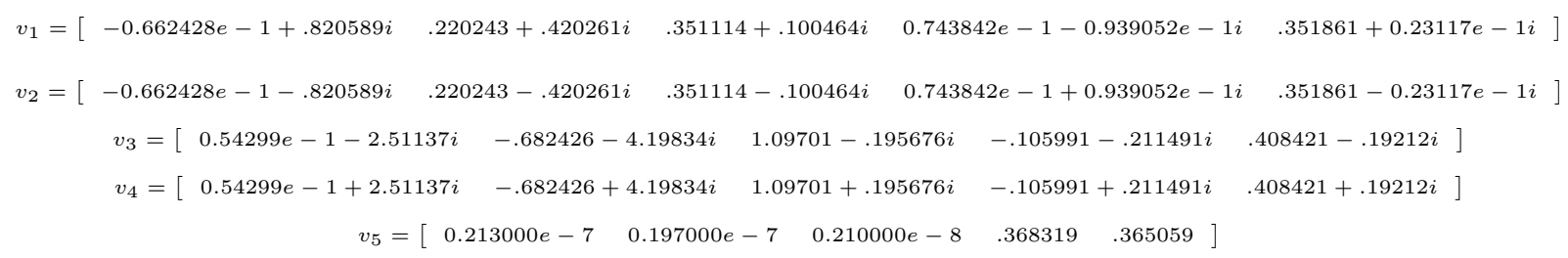

The unstable manifold $M_{u}$ is generated by the vectors $\left\langle v_{1}, v_{2}, v_{5}\right\rangle$ while the stable manifold $M_{s}$ by the vector $\left\langle v_{3}, v_{4}\right\rangle$. Notice that in this example only one eigenvalue is real.

The previous two examples have been implemented using MAPLE 13. They show that the economy converges to its steady state through a saddle path and the unstable transitional manifold has dimension three while the stable manifold has dimension two.

Along the BGP, the consumption-capital ratio is an increasing function of the inverse of the elasticity of substitution, $\sigma$, and of the rate of time preference, $\rho$; it, instead, depends negatively on the capital share, $\alpha$. Notice that it is independent of the human capital share, $\beta$. The stationary share of human capital allocated to physical and knowledge production instead are functions of the physical and human capital shares, $\alpha$ and $\beta$, of the elasticity of human capital in the knowledge production process, $\phi$, of the rate of time preference, $\rho$ and of the inverse of the intertemporal elasticity of substitution in consumption, $\sigma$. In particular, the share of human capital allocated to knowledge (physical) production is a decreasing (increasing) function of $\beta$ and an increasing (decreasing) function of $\phi$; the share allocated to the educational sector instead is independent of both $\sigma$ and $\phi$. 


\subsection{The Allocation of Human Capital}

We now illustrate the behavior of the allocation of human capital among the three sectors implied by the model, under a given set of parameter values. In choosing such values we rely on existing empirical estimates or on baseline specifications coming from previous works.

The physical capital share has been traditionally considered to be around one third (see Denison, 1962; Maddison, 1982; Jorgenson et al., 1987; Mankiw et al., 1992) while the human capital share has been estimated by Mankiw, Romer and Weil (1992) to vary in the range $(0.333,0.5)$. The elasticity of ideas in the production function of technology has been estimated by Kortum (1993) in the range (0.1, 0.6), implying that $\phi \in(0.4,0.9)$. We set $\alpha=0.33$, and following Mulligan and Sala-i-Martin (1993), $\rho=0.04$ and $\sigma=2$. Firstly, we fix $\beta=0.42$ (the median value of the interval estimated by Mankiw, Romer and Weil, 1992) and let $\phi$ vary in the interval $(0.4,0.9)$; then we fix $\phi=0.65$ (the median value of the interval estimated by Kortum, 1993) and let $\beta$ vary in the range $(0.333,0.5)$.

This set of parameters implies that in steady state the highest share of human capital is devoted to creation of new human capital, and the lowest share is allocated to knowledge production. Such an outcome is clear: the impact of human capital is more important in the educational sector, since it is the growth driven force, and in the physical one, since it produces the consumable good, which is the argument of agents' utility function, while it is lower in the technological sector. This ranking is clearly reflected by the optimal allocation of resources. In fact, the share of human capital in the technological sector is always particularly low, while that in physical production can reach a value close to that in education.
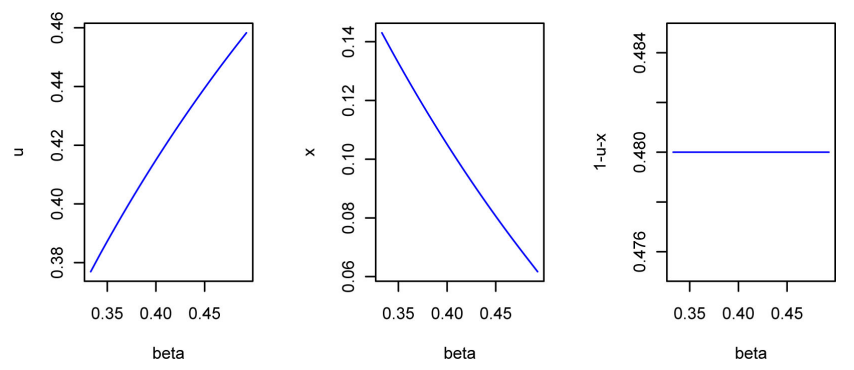

Figure 2: Optimal allocation of human capital in steady state. The figure shows the values of $u, x$ and $1-u-x$, as $\beta$ changes

Notice that even if we let $\beta$ and $\phi$ be in the ranges empirically estimated, the same result holds. Of course, as one of these parameters changes, the steady state values of $u$ and $x$ changes, while that of $1-u-x$ does not. If we increase $\beta, u$ increases (and $x$ decreases) but its steady state value is always lower than the share allocated to the educational sector; in fact, when $\beta=0.5$, that is the upper bound of its estimated range, the optimal allocation of human capital is the following: $u^{*}=0.46, x^{*}=0.06$ and $1-u^{*}-x^{*}=0.48$. Only if the human capital share gets particularly large (higher than 0.56 ), the fraction of human capital allocated to physical production will be higher than that in education.

If we increase $\phi$, instead, $x$ increases (and $u$ decreases) but, again, the steady state value of $u$ and $x$ are always lower than the share allocated to educational sector; in fact, when $\phi=0.4$, the lower bound of its estimated range, the optimal allocation of human capital is: $u^{*}=0.44, x^{*}=0.07$ and $1-u^{*}-x^{*}=0.48$. If $\phi$ gets particularly small (lower than 0.2 ), then the fraction of human capital allocated to physical production will be higher than that in education.

\section{Conclusions}

During the last century, knowledge (technology) has increased consistently in most of the industrialized countries. Economic growth theory introduces endogenous technical change in order to describe this fact. 

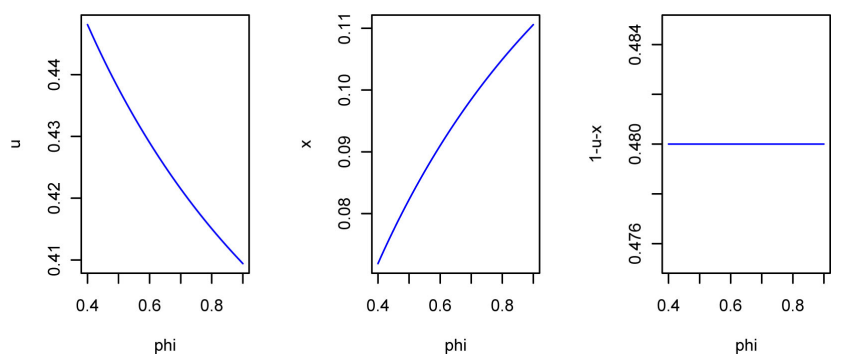

Figure 3: Optimal allocation of human capital in steady state. The figure shows the values of $u, x$ and $1-u-x$, as $\phi$ changes

As a result, a new literature stream has recently arisen, leading to ideas-based growth models. Our goal is to introduce ideas in a standard multi-sector endogenous growth model and the natural candidate for such an aim seems to be the Uzawa-Lucas model. Therefore, we extend it along different lines: we formally introduce ideas, which are used for producing the final physical good and are created in a particular separate sector, and we emphasize the importance of education in the process generating ideas, also considering the endogenous allocation of human capital in this technical sector. We also consider the effect of population dynamics and public expenditure. The interaction between human capital and ideas rules the economy. Since human capital is a rival good, while ideas are not, the allocation of human capital across sectors is crucial. The stock of ideas can be contemporaneously exploited in the final and technical sectors, while human capital stock has to be shared across the three sectors. Therefore, the planner has to determine how to optimize the trade-off arising from the allocation of resources.

We show that the economy admits a steady state equilibrium, along which the consumption-capital ratio is constant. The stable and unstable manifolds are multidimensional, and the unstable manifold is at least of dimension one. It remains an open problem if it is possible to obtain, maybe for a certain combination of parameters, a closed-form solution to this model.

For further research, we suggest to focus on the decentralized outcome, investigating how this allocation differs from the optimal planned one and how it can be possible to decentralize the optimal allocation of resources. Another aspect which could deserve some attention is the production function of ideas: we assume it is neoclassical; however, it could be interesting to analyze the case where it is somehow linear, meaning that ideas represent an additional source of endogenous growth. Another interesting extension of this paper could involve a spatial Uzawa-Lucas model by assuming a continuous space structure and nonconcave production functions (see, for instance, Boucekkine and others (2009a) and Capasso and others (2010a and 2010c)).

\section{A Steady State}

The steady state of the five dynamical equations system is characterized by setting equations (26), (27), (28), (29) and (30) equal to zero:

$$
\begin{aligned}
0 & =\frac{\alpha-\sigma}{\sigma} Z^{*}-\frac{\rho}{\sigma}+\chi^{*} \\
0 & =M^{*}-Z^{*}+\chi^{*} \\
0 & =1-u^{*}-x^{*}-Z^{*}+\chi^{*} \\
0 & =\frac{1}{\beta-1}\left[\alpha \chi^{*}-\beta-(1-\beta)\left(u^{*}+x^{*}\right)-(1-\alpha-\beta) M^{*}\right] \\
0 & =\frac{1}{\phi-1}\left[\frac{\phi(1-\alpha-\beta)}{\beta} \frac{u^{*}}{x^{*}} M^{*}-\phi-(1-\phi)\left(u^{*}+x^{*}\right)\right] .
\end{aligned}
$$


Plugging equation (39) into (38) we get:

$$
1-u^{*}-x^{*}=M^{*}
$$

substituting equation (42) into equation (40), we obtain:

$$
\chi^{*}=\frac{1}{\alpha}-M^{*}
$$

Substituting equation (43) into (37) instead:

$$
Z^{*}=\frac{\sigma}{\alpha} M^{*}+\frac{\rho}{\alpha} .
$$

Then, from equations (38) and (44) we obtain the steady state value of $M$ :

$$
M^{*}=\frac{1-\rho}{\sigma} .
$$

Therefore those of $\chi$ and $Z$ are:

$$
\begin{aligned}
& \chi^{*}=\frac{\sigma-\alpha(1-\rho)}{\alpha \sigma} \\
& Z^{*}=\frac{1}{\alpha} .
\end{aligned}
$$

From equation (41), we have:

$$
u^{*}=\frac{\beta \phi(1-\rho)+\beta(\sigma+\rho-1)}{\phi(1-\alpha-\beta)(1-\rho)} x^{*},
$$

which substituted in equation (42) yields:

$$
x^{*}=\frac{\phi(1-\alpha-\beta)(1-\rho)(\sigma+\rho-1)}{\sigma[\phi(1-\alpha)(1-\rho)+\beta(\sigma+\rho-1)]}
$$

and therefore:

$$
u^{*}=\frac{(\sigma+\rho-1)[\beta \phi(1-\rho)+\beta(\sigma+\rho-1)]}{\sigma[\phi(1-\alpha)(1-\rho)+\beta(\sigma+\rho-1)]}
$$

Notice that the steady state values of the five variables, given by expressions (45), (46), (47), (49) and (50), are positive if the following conditions hold:

$$
\left.\begin{array}{c}
1-\rho>0 \rightarrow \rho<1 \\
\sigma-\alpha(1-\rho)>0 \\
\sigma+\rho-1>0
\end{array}\right\} \rightarrow \sigma>(1-\rho)>\alpha(1-\rho)
$$

\section{B Local Stability}

We can study the stability of the steady state, by linearizing the system of differential equations. The Jacobian matrix, $J\left(\chi_{t}, Z_{t}, M_{t}, u_{t}, x_{t}\right)$, is:

$$
\left[\begin{array}{cc}
\frac{\alpha-\sigma}{\sigma} Z_{t}-\frac{\rho}{\sigma}+2 \chi_{t} & \frac{\alpha-\sigma}{\sigma} \chi_{t} \\
\frac{\alpha+\beta-1}{\beta-1} Z_{t} & \frac{\alpha+\beta-1}{\beta-1} \chi_{t}-\frac{\beta}{\beta-1}-2(1-\alpha) Z_{t}-\frac{1-\alpha-\beta}{\beta-1} M_{t} \\
0 & 0 \\
\frac{\alpha}{\beta-1} u_{t} & 0 \\
0 & 0
\end{array}\right]
$$




$$
\begin{aligned}
& {\left[\begin{array}{cc}
0 & 0 \\
-\frac{1-\alpha-\beta}{\beta-1} Z_{t} & 0 \\
\frac{2 \phi^{2}(1-\alpha-\beta)}{\beta(\phi-1)} \frac{u_{t}}{x_{t}} M_{t}-2 \phi M_{t}-\frac{\phi}{\phi-1} & \frac{\phi^{2}(1-\alpha-\beta)}{\beta(\phi-1)} \frac{M_{t}^{2}}{x_{t}} \\
-\frac{1-\alpha-\beta}{\beta-1} u_{t} & \frac{1}{\beta-1}\left[\alpha \chi_{t}-\beta-(1-\beta)\left(2 u_{t}+x_{t}\right)-(1-\alpha-\beta) M_{t}\right] \\
\frac{\phi(1-\alpha-\beta)}{\beta(\phi-1)} u_{t} & \frac{\phi(1-\alpha-\beta)}{\beta(\phi-1)} M_{t}+x_{t} \\
0 \\
0 \\
{\left[\begin{array}{c}
\beta(\phi-1) \\
u_{t}
\end{array}\right]} \\
\frac{1}{x_{t}^{2}} M_{t}^{2}\left[-\phi-(1-\phi)\left(u_{t}+2 x_{t}\right)\right]
\end{array}\right],}
\end{aligned}
$$

and, evaluated at the steady state, $J\left(\chi^{*}, Z^{*}, M^{*}, u^{*}, x^{*}\right)$, it can also be written as:

$$
\left[\begin{array}{ccccc}
\chi^{*} & \frac{\alpha-\sigma}{\sigma} \chi^{*} & 0 & 0 & 0 \\
\frac{\alpha+\beta-1}{\beta-1} Z^{*} & -(1-\alpha) Z^{*} & -\frac{1-\alpha-\beta}{\beta-1} Z^{*} & 0 & 0 \\
0 & 0 & \frac{\phi^{2}(1-\alpha-\beta)}{\beta(\phi-1)} \frac{u^{*}}{x^{*}} M^{*}-\phi M^{*} & \frac{\phi^{2}(1-\alpha-\beta)}{\beta(\phi-1)} \frac{\left(M^{*}\right)^{2}}{x^{*}} & -\frac{\phi^{2}(1-\alpha-\beta)}{\beta(\phi-1)} \frac{u^{*}}{\left(x^{*}\right)^{2}}\left(M^{*}\right)^{2} \\
\frac{\alpha}{\beta-1} u^{*} & 0 & -\frac{1-\alpha-\beta}{\beta-1} u^{*} & u^{*} & u^{*} \\
0 & 0 & \frac{\phi(1-\alpha-\beta)}{\beta(\phi-1)} u^{*} & \frac{\phi(1-\alpha-\beta)}{\beta(\phi-1)} M^{*}+x^{*} & x^{*}-\frac{\phi(1-\alpha-\beta)}{\beta(\phi-1)} \frac{u^{*}}{x^{*}} M^{*}
\end{array}\right]
$$

that is:
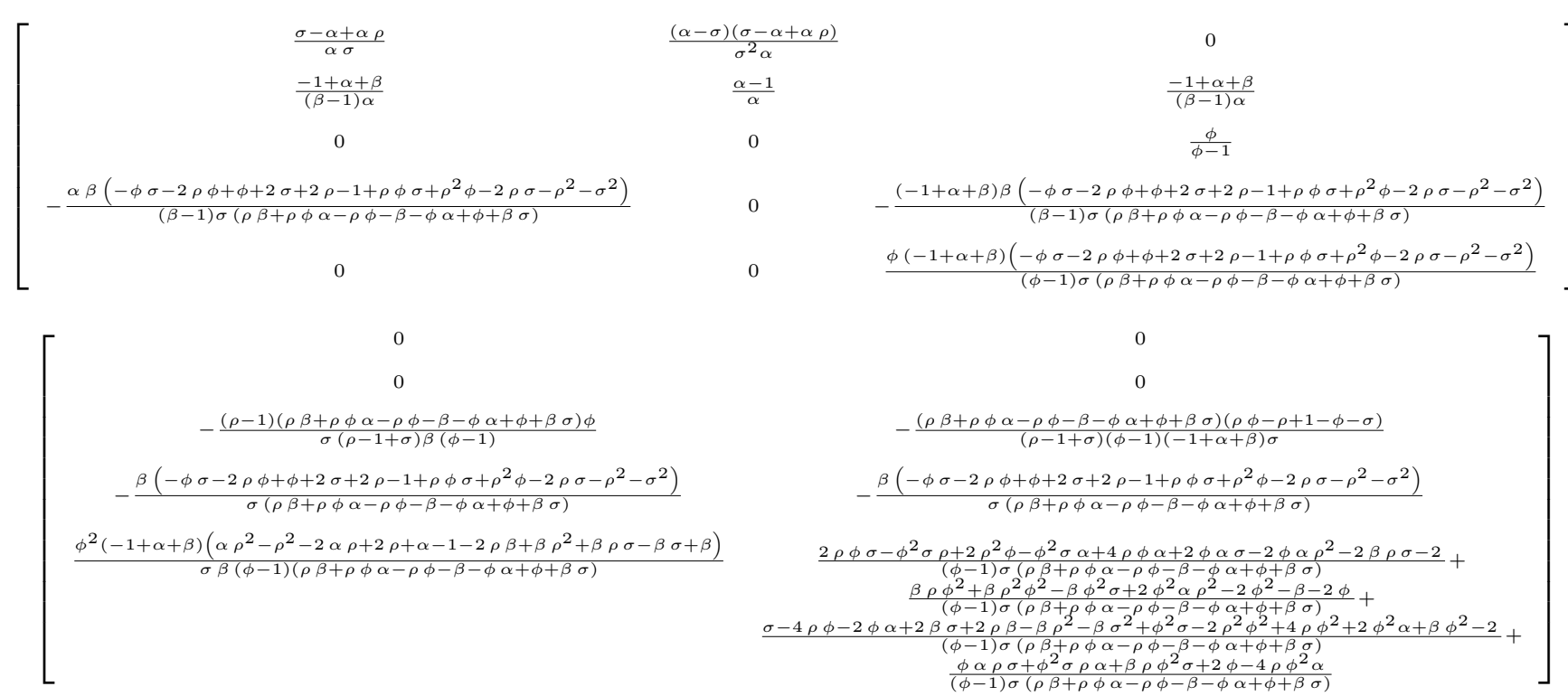

Remembering that the sum of the eigenvalues of a matrix is equal to the trace of the matrix, we can study the sign of the trace of the Jacobian evaluated at steady state: if it is positive, this would mean that at least one eigenvalue is positive. The trace of $J\left(\chi^{*}, Z^{*}, M^{*}, u^{*}, x^{*}\right)$ is:

$$
\operatorname{tr}\left(J\left(\chi^{*}, Z^{*}, M^{*}, u^{*}, x^{*}\right)\right)=\frac{3(\sigma+\rho-1)}{\sigma},
$$

which is positive if:

$$
\sigma>(1-\rho)
$$

Notice that the same condition ensures that the steady state values of the variables are positive. This means that, considering the standard empirical value of $\rho$, about $0.04, \sigma$ has to be higher than 0.96 . This does not represent a unreasonable value for the inverse of the elasticity of substitution in consumption, as empirical 
works suggest (see for example, Mehra and Prescott, 1985; and Hall, 1988). If condition (54) holds, there exists at least one positive eigenvalue.

Moreover, it is possible to show that the determinant of the Jacobian, $\operatorname{det}\left(J\left(\chi^{*}, Z^{*}, M^{*}, u^{*}, x^{*}\right)\right)$, which equals the product of the eigenvalues, is:

$$
-\frac{(\alpha-1)(\rho-1+\sigma)(\rho-1)((\phi-1) \rho+1-\phi-\sigma) \phi(\sigma-\alpha+\alpha \rho)}{(\phi-1)(\beta-1) \alpha \sigma^{4}} .
$$

Under the condition $\sigma>1-\rho$ it is easy to prove that this determinant is positive. Since the product of the eigenvalues is positive and their sum is positive (under the condition $\sigma>1-\rho$ ), if the eigenvalues are real numbers then the number of positive eigenvalues has to be odd, that is there can be one or three or five positive eigenvalues (and consequently, four or two or zero negative eigenvalues). Therefore an unstable transitional manifold of at least dimension one exists.

\section{References}

[1] Barro R.J. and Lee J.W. (2000), International Data on Educational Attainment: Updates and Implications, CID Working Paper N. 42.

[2] Barro R.J. (1990), Government Spending in a Simple Model of Endogenous Growth, Journal of Political Economy, 98 (5), pp. 103-126.

[3] Barro R.J. and Sala-i-Martin X. (1992), Public Finance in Models of Economic Growth, Review of Economic Studies, 59 (4), pp. 645-661.

[4] Boucekkine R., Martinezz B. and Ruiz-Tamarit J.R. (2009b), The effect of population growth in a two-sector growth model, Working Paper, Université Catholique de Louvain.

[5] Boucekkine R., Camacho C. and Zou B.(2009a), Bridging the gap between growth theory and the new economic geography: the spatial Ramsey model, Macroeconomic Dynamics 13, pp. 2045.

[6] Boucekkine R., Martinez B. and Ruiz-Tamarit J.R. (2008b), A Note on Global Dynamics and Imbalance Effects in the Lucas-Uzawa Model, International Journal of Economic Theory 4, pp. 503-518.

[7] Boucekkine R. and Ruiz-Tamarit J.R. (2008a), Special Functions for the Study of Economic Dynamics: The Case of the Lucas-Uzawa Model, Journal of Mathematical Economics 44, pp. 33-54;

[8] Bucci A., Kunze H. and La Torre D. (2010a), Embodied Technical Change and Learning-by-Doing in a Two-Sector Growth Model with Human Capital Accumulation, in Optimal Control of Age-structured Populations in Economy, Demography, and the Environment (R. Boucekkine, N. Hritonenko Eds.), Routledge (Taylor \& Francis, UK), Environmental Economics Series.

[9] Bucci A., Kunze H. and La Torre D. (2010b), Parameter Identification, Population and Economic Growth in an Extended Lucas and Uzawa-type Two Sector Model, Nonlinear Studies, in press.

[10] Bucci A., Colapinto C., Forster M. and La Torre D. (2010c), On human capital and economic growth with random technology shocks, Working Paper N. 2008-36, Department of Economics, Business and Statistics, University of Milan. Presented during the First Workshop on Dynamics, Optimal Growth and Population Change: Theory and Applications, 18/19-09-2008, Milan.

[11] Bucci A., Florio M. and La Torre D. (2010d), Transitional dynamics in a growth model with government spending, technological progress and population change, Working Paper N. 2009-38, Department of Economics, Business and Statistics, University of Milan. Presented during the First Workshop on Dynamics, Optimal Growth and Population Change: Theory and Applications, 18/19-09-2008, Milan. 
[12] Bucci A. and La Torre D. (2009), Population and economic growth with human and physical capital investments, International Review of Economics 56 (1), pp. 17-27.

[13] Capasso V., Engbers R. and La Torre D. (2010a), On a spatial Solow-model with technological diffusion and nonconcave production function, Nonlinear Analysis Real World Applications, in press.

[14] Capasso V., Kunze H. and La Torre D. (2010b), Population dynamics in growth models with convexconcave production functions and migration effects, Proceedings of the MAF Conference, Ravello, 4-8 April, 2010.

[15] Capasso V., Engbers R. and La Torre D. (2010c), Population dynamics in a spatial Solow model with convex-concave production functions, Proceedings of the MAF Conference, Ravello, 4-8 April, 2010.

[16] Chilarescu C. (2008), An analytical solutions for a model of endogenous growth, Economic Modelling 25 , pp. $1175-1182$.

[17] Denison E.F. (1962), Sources of Growth in the United States and the Alternatives Before Us, Supplement Paper 13, New York: Committee for Economic Development.

[18] Ferrara M. and Guerrini L. (2010), A note on the Uzawa-Lucas model with unskilled labor, Applied Sciences 12, pp.90-95.

[19] Ferrara M. and Guerrini L. (2008), On the dynamic of three sector growth model, International Review of Economics 55 (3), pp.275-284.

[20] Hall R.E. (1988), Intertemporal Substitution in Consumption, Journal of Political Economy 96 (2), pp. 339-357

[21] Hiraguchi R. (2009a), A solution to the Lucas-Uzawa model with increasing returns to scale: note, Economic Modelling, in press, doi:10.1016/j.econmod.2009.02.001.

[22] Hiraguchi R. (2009b), Non-concavity problems in the dynamic macroeconomic models: a note, Journal of Banking and Finance 33, pp. 568-572.

[23] Kunze H.E. and La Torre D. (2009b), Solving inverse problems for differential equations by the collage method and application to an economic growth model International Journal of Optimization: Theory Methods and Applications 1 (1), pp. 26-35.

[24] Kunze H., La Torre D. and Vrscay E.R. (2009a), Inverse problems for random differential equations using the collage method for random contraction mappings, Journal of Computational and Applied Mathematics 223 (2), pp. 853-861.

[25] Kunze H., La Torre D., Vrscay E.R. (2007a), Random fixed point equations and inverse problems using collage method for contraction mappings, Journal of Mathematical Analysis and Applications 334, pp. 1116-1129.

[26] Kunze H., La Torre D., Vrscay, E. R. (2007b), Solving inverse problems for DEs by collage method and applications to variational optimization, Proceedings of the Symposium on Inverse Problems, Design and Optimization (S.Dulikravich, M.J.Colaco,H.R.B.Orlande,M.Tanaka eds.), Miami, April 16-18, pp. 533-539.

[27] Kunze H., La Torre D. and Vrscay E.R. (2007c), Solving inverse problems for random equations and applications, Proceedings of the Symposium on Inverse Problems, Design and Optimization (S.Dulikravich, M.J.Colaco,H.R.B.Orlande,M.Tanaka eds.), Miami, April 16-18, pp. 540-547. 
[28] Kunze H., Hicken J. and Vrscay E.R. (2004), Inverse problems for ODEs using contraction functions: suboptimality of the Collage Method, Inverse Problems 20, pp. 977-991.

[29] Kunze H. and Gomes S. (2003), Solving an inverse problem for Urison-type integral equations using Banach's fixed point theorem, Inverse Problems 19, pp. 411-418.

[30] Kunze H. and Vrscay, E.R. (1999), Solving inverse problems for ordinary differential equations using the Picard contraction function, Inverse Problems 15, pp. 745-770.

[31] Jones C.I. (1995), Time Series Test of Endogenous Growth Models, Quarterly Journal of Economics 110 (441), pp. 495-525.

[32] Jones C.I. (2005), Growth and Ideas, in Aghion P. and Durlauf S. eds, Handbook of Economic Growth (North-Holland: Amsterdam)

[33] Jorgenson D.W., Gollop F.M. and Fraumeni B.M. (1987), Productivity and U.S. Economic Growth, (Cambridge, MA: Harvard University Press).

[34] Kortum S.S. (1993), Equilibrium RESD and the Patent REDD Ratio: U.S. Evidence, American Economic Review 83 (2), pp. 450-45.7

[35] Lotka A.J. (1925), Elements of physical biology, Baltimore: Williams and Wilkins.

[36] Lucas R.E. (1988), On the Mechanics of Economic Development, Journal of Monetary Economics 22, pp. 3-42.

[37] Lutz G. A. (1998), Growth, Welfare, and Trade in an Integrated Model of Human-Capital Accumulation and Research, Journal of Macroeconomics 20 (1), pp. 81105.

[38] Maddison A. (1982), Phases of Capitalist Development, (Oxford: Oxford University Press).

[39] Malthus T.R. (1798), An Essay on the Principle of Population, as It Affects the Future Improvement of Society with Remarks on the Speculations of Mr. Godwin, M. Condorcet, and Other Writers. London: J. Johnson (First Edition).

[40] Mankiw G. N., Romer D. and Weil D. N. (1992), A Contribution to the Empirics of Economic Growth, Quarterly Journal of Economics 107 (2), pp. 407-437.

[41] Mehra R. and Prescott E. (1985), The Equity Premium: a Puzzle, Journal of Monetary Economics 15, pp. $145-161$.

[42] Pessoa A. (2005), Ideas" Driven Growth: the OECD Evidence, Portuguese Economic Journal 4 (1), pp. 46-67.

[43] Romer P.M. (1986), Increasing Returns and Long-Run Growth, Journal of Political Economy 94, pp. 1002-1037.

[44] Romer P.M. (1990), Endogenous Technological Change, Journal of Political Economy 98 (5), pp. S71S102.

[45] Romer P.M. (1993), Two Strategies for Economic Development: Using Ideas and Producing Ideas, Proceedings of the World Bank Annual Conference on Development Economics 1992, pp. 63-115.

[46] Stokey N.L. (1995), RESD and Economic Growth, Review of Economic Studies 62 (3), pp. 469-489. 
[47] Uzawa H. (1965), Optimum Technical Change in an Aggregative Model of Economic Growth, International Economic Review 6, pp. 18-31.

[48] Verhulst P. (1977), A note on the law of population growth, in D. Smith and N. Keyfitz (eds.): "Mathematical Demography. Selected papers". Springer, Berlin.

[49] Volterra V. (1931), Variations and fluctuations of the number of individuals in animal species living together, in "Animal Ecology", McGraw-Hill. 